Yet there remains ongoing debate as to whether such models offer advantages over routine clinical services. As such, there has been low uptake of community-based HIV testing in some countries. To better understand the processes and outcomes of these programs to inform future implementation, we systematically reviewed published studies.

Methods We searched Medline, EMBASE and Cochrane databases from 1980 to October 2010. Included studies described HIV testing outcomes of community based testing services that included gay men as clients. The primary outcomes were client testing history and HIV positivity.

Results We identified 33 papers that described 44 community-based HIV testing services. There were 18 on-site only services (community based organisations/community clinics, including one multi-fixed site), seven on-site services with outreach and 19 outreach only services, including eight outreach services in venues (bar, club, sauna); six mobile testing facilities and five community outreach sites in multiple locations (See Abstract P5-S7.16 table 1). The majority of the services were in the US (28 of 44) and 34 of 44 offered rapid HIV antibody testing on-site at the point-of-care. OraQuick Advance Rapid HIV-1/2 Antibody or Abbott Determine HIV-1/2 rapid with finger-stick were the most common tests used. Among services reporting testing outcomes specifically for gay men (22 of 44), the median proportion of men who had never tested for HIV prior to attending the community-based testing service was $34.1 \%$ (range: $7.8 \%-44.0 \%$ per service). The median HIV positivity was $3.9 \%$ (range: $0.3 \%-60.0 \%$ per service) and the median return rate for confirmatory testing was $83.8 \%$ (range: $22.7 \%-95.0 \%$ per service); higher in community-based organisation services $(84.2 \%)$ and lower in outreach models (33.0\%).

Conclusion Community-based HIV testing services provide a model of HIV testing that attracts a significant proportion of gay men who have never tested before, and these men are at high risk of HIV as evidenced by the HIV positivity rate.

\section{P5-S7.17 ACCEPTING THE GOOD WITH THE BAD: “BARRIERS AND FACILITATORS OF COMMUNITY-BASED HIV TESTING SERVICES FOR GAY MEN: A SYSTEMATIC REVIEW"}

doi:10.1136/sextrans-2011-050108.612

${ }^{1} \mathrm{~A}$ Pedrana, ${ }^{1} \mathrm{M}$ Stoove, ${ }^{1} \mathrm{~A}$ Bowring, ${ }^{1} \mathrm{M}$ Hellard, ${ }^{2} \mathrm{R}$ Guy. ${ }^{1}$ Burnet Institute, Melbourne, Australia; ${ }^{2}$ National Centre in HIV Epidemiology and Clinical Research Sydney Australia

Background With a global focus on increased HIV testing among high risk groups including men who have sex with men (MSM), many community-based HIV testing services have been established in recent years with the goal of increasing testing opportunities for populations at risk. To better understand the acceptability of community based HIV testing models targeting MSM from the provider and consumer perspective we systematically reviewed published studies.

Methods We searched Medline, EMBASE and Cochrane databases from 1980 to October 2010. Studies were included if they described acceptability of community based HIV testing services targeting MSM, including outreach settings (eg, saunas, public events), collected through surveys, in-depth interviews, focus groups, or exit forms. A quantitative descriptive analysis of the barriers and facilitators of community based HIV testing identified by service providers and consumers was conducted.

Results We identified 25 papers that met our selection criteria and were included in the review see Abstract P5-S7.17 table 1. Twenty one studies focused on facilitators from the consumers' perspective, with testing convenience, provision of rapid testing, and acceptability/feeling comfortable with settings reported at factors that facilitated seeking HIV testing at community based services. From the provider perspective (six studies) key factors enhancing service acceptability were client friendly protocols, service promotion, offering additional clinical services, and effective protocols for follow-up and referral. Sixteen studies captured barriers to using community based HIV testing services from the consumer perspective and the main issue reported related to readiness to receive results on the same day or in the community-based environment. Providers in six studies reported difficulties in followup, testing in outreach settings, cost, providing adequate staff training, managing workload and developing and maintaining referral pathways as key barriers.

Conclusion Acceptability from both consumers and service providers is important to ensure an efficient and sustainable service. The experiences of many other services collated in this review will help other organisations address potential barriers and facilitators to the implementation of community-based HIV testing services

Abstract P5-S7.17 Table 1 Summary of community HIV testing services (sample size) $(n=25)$

\begin{tabular}{|c|c|c|}
\hline Category & Subcategory & All services $\mathbf{n}(\%)$ \\
\hline \multirow[t]{7}{*}{ Location } & Canada & $1(4.0)$ \\
\hline & Hong Kong & $1(4.0)$ \\
\hline & New Zealand & $1(4.0)$ \\
\hline & Switzerland & $1(4.0)$ \\
\hline & The Netherlands & $3(12.0)$ \\
\hline & UK & $3(12.0)$ \\
\hline & USA & $15(60.0)$ \\
\hline \multirow[t]{3}{*}{ Target group } & MSM & $15(60.0)$ \\
\hline & Multiple & $7(28.0)$ \\
\hline & Unclear - CBO clients & $3(12.0)$ \\
\hline \multirow[t]{6}{*}{ Services type* } & $\mathrm{CBO} /$ community centre & $10(40.0)$ \\
\hline & STD clinic & $3(12.0)$ \\
\hline & Mobile testing facility & $1(4.0)$ \\
\hline & Needle exchange programs & $2(8.0)$ \\
\hline & $\begin{array}{l}\text { Venue-based outreach (bar, } \\
\text { club, sauna) }\end{array}$ & $9(36.0)$ \\
\hline & $\begin{array}{l}\text { Combination (eg, } \mathrm{CBO} \text {, outreach, } \\
\text { mobile testing facility). }\end{array}$ & $5(20.0)$ \\
\hline \multirow{4}{*}{$\begin{array}{l}\text { Rapid testing } \\
\text { offered }\end{array}$} & Yes & $13(52.0)$ \\
\hline & No & $5(20.0)$ \\
\hline & $\begin{array}{l}\text { Yes }- \text { in combination with } \\
\text { standard testing }\end{array}$ & $4(16.0)$ \\
\hline & Unclear & $3(12.0)$ \\
\hline \multirow[t]{7}{*}{ Study design } & Case studies & $1(4.0)$ \\
\hline & Clinical audit & $1(4.0)$ \\
\hline & Cross-sectional studies & $4(16.0)$ \\
\hline & Evaluation reports & $4(16.0)$ \\
\hline & Pilot/feasibility studies & $4(16.0)$ \\
\hline & Qualitative studies & $10(40.0)$ \\
\hline & Randomised trails (RCT) & $1(4.0)$ \\
\hline \multirow{5}{*}{$\begin{array}{l}\text { Data collection } \\
\text { methods* }\end{array}$} & Client surveys (including exit forms) & $15(60.0)$ \\
\hline & Focus groups & $2(8.0)$ \\
\hline & In-depth interviews & $5(20.0)$ \\
\hline & Provider surveys & $2(8.0)$ \\
\hline & Qualitative phone interviews & $3(12.0)$ \\
\hline \multirow[t]{3}{*}{ Study participants } & Clients & $17(68.0)$ \\
\hline & Providers & $4(16.0)$ \\
\hline & Clients \& providers & $4(16.0)$ \\
\hline
\end{tabular}

${ }^{*}$ Service types and data collection methods are not mutually exclusive, so \% do not add to $100 \%$ 